\title{
Integration of Optical Telecommunications and Radio Access Networks to Assure Quality of Service
}

\author{
Marco Giuntini, Jessica Morabito, Alessandro Valenti, Francesco Matera, \\ Valeria Carrozzo*, Silvia Di Bartolo* \\ Fondazione Ugo Bordoni, via B. Castiglione 59, 00142 Rome, Italy \\ *Istituto Superiore delle Comunicazioni e Tecnologie dell'Informazione \\ viale America 201, 00144 Rome, Italy \\ Email:avalenti@fub.it
}

\begin{abstract}
In this work, we show how to control a Quality of Service in optical networks integrated with WiMAX access radio by means of the MPLS and WiMAX Class of Services, even in condition of traffic congestions.

Keywords: Optical Networks, WiMAX, EPON, WDM PON, QoS.

\section{INTRODUCTION}

The growth of high bit-rate services, such as TV over IP (IPTV and High Definition IPTV [1]), Web-TV (e.g. YouTube), and also the increasing popularity of social networks, resulting in a multimedia content exchange, require to current telecommunications networks a migration towards architectures and technologies capable of ensuring ever higher bandwidth. This adjustment must be made in all sections of the network, especially in the access network where the bandwidth limitations bottle up the next-generation networks development.

In this scenario, it is clear that optical fibre deployment is fundamental for future metro-access networks and, among Fibre To The X (FFTX) architectures, Passive Optical Networks (PONs) are the simplest and cheapest to implement [2]. However, in some rural areas, where the installation of optical fibres would be too expensive or even not feasible, fixed radio access technologies, as WiMAX [3], result to be of main importance to have high bandwidth connections [4]. Due to this fact, in the near future, integration of optical access network with radio access ones can be seen as solution against digital divide, but some issues have to be addressed. In particular, in the radio access networks, where bandwidth is shared among all users, Quality of Service (QoS) assumes a key importance role, requiring the identification of techniques that, in case of congestions or possible bottlenecks in the different network sections, are able to meet performance requirements of different services.

This paper proposes an "end-to-end" QoS management (i.e. from source to destination) in an optical core network with WiMAX radio access. The tests are carried out by OPNET simulator and mainly focused on three architectural scenarios where the Base Station (BS) is connected: (a) directly to a node of the section edge of the considered network, (b) to an Optical Network Unit (ONU) of a (TDM) PON, (c) to a ONU of a WDM-PON. These choices allow simulating various scenarios for integration of a WiMAX access network, and therefore different situations in which assure QoS.
\end{abstract}

\section{STANDARD IEEE 802.16 - WIMAX}

WiMAX is a technology for wireless transmission of broadband access. It can be used, as other wireless technologies, on many areas (from urban to rural environments). The technology supports data shared transmission speeds up to 70Mbit/s and up to 5 types of Classes of Service (CoS), from the Unsolicited Grant Service (UGS) for real-time systems with fixed size, to the Best Effort (BE) for data streams where undue least level of service [4].

\section{TECHNIQUES TO MANAGE QOS IN CORE AND EDGE NETWORKS}

During the migration from current networks to the Next Generation Networks (NGN), in all sections of the network there will be a transition towards packet switched paradigm, and Multiprotocol Label Switching (MPLS) represents one of candidate techniques to be used in the core network as packet transport technology. Among the advantages of this technique, there is certainly an efficient management of QoS, defining logical paths, Label Switched Paths (LSP), with a specific CoS. In particular, it can be defined up to eight CoSs using the Traffic Class (TC) field of the MPLS label.

In the edge network, Ethernet technology can be the next technology to employ due to its characteristics as simplicity and cheapness. In this case, the QoS is managed by means of Virtual LANs (VLAN), in particular by 3 bits in the VLAN Tag User Priority field, with a maximum of eight CoSs.

In this way, the QoS is managed end-to-end by mapping of CoSs defined in the VLAN and those defined in the LSP.

\section{PRELIMINARY TEST: TEST-BED WITH WIMAX ACCESS}

In this case, a BS is connected to the optical network to an EDGE point (Fig. 1). As example, it was reproduced the core-edge network representing the ISCTI-FUB test-bed (consisting of four routers). 
In the simulation, four Best Effort IP flows are generated from Web Server to terminals SS_1, SS_3, SS_4, SS_5. Another IP flows (Video Stream with high priority CoS) from the Video Server to the terminal SS_2 is generated after 5 mins; such IP flow carries a Video Stream of about $20 \mathrm{Mbit} / \mathrm{s}$ and it is treated with guaranteed QoS by means of: VLAN Tagging between Video Server and router R1, LSP in the core network, and Classes of Service in WiMAX cell. Fig. 2 shows the traffic received from the WiMAX terminals. It can be observe that, when the terminals receives Video Stream flow, only the throughput of the Web flows experiences degradation because of the congestion in the WiMAX cell; this is due to bandwidth reservation performed by the BS to the terminal receiving the Video Streaming.

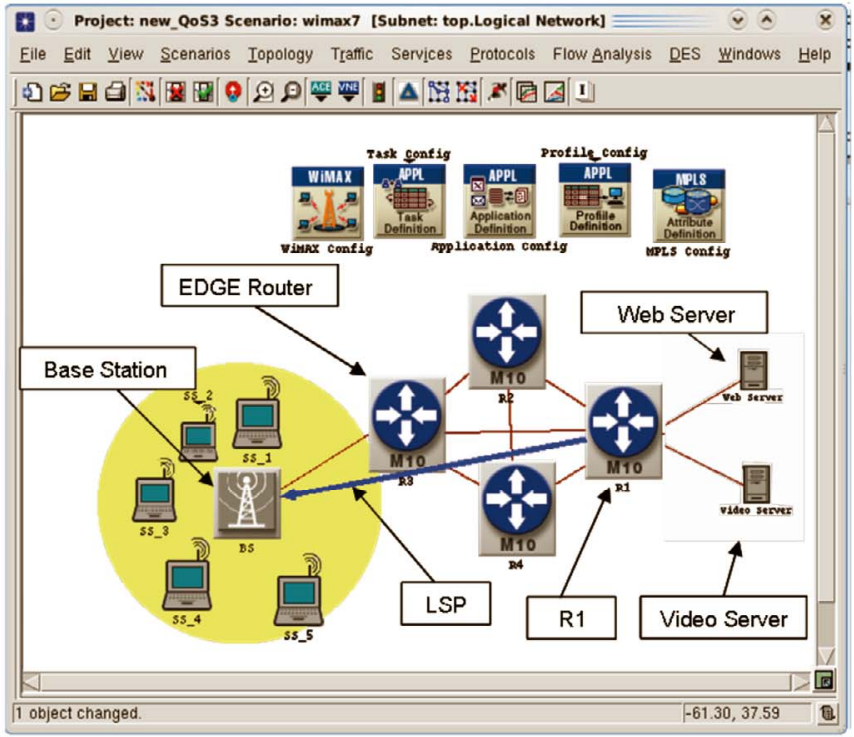

Figure 1. Base Station connected to an EDGE point.

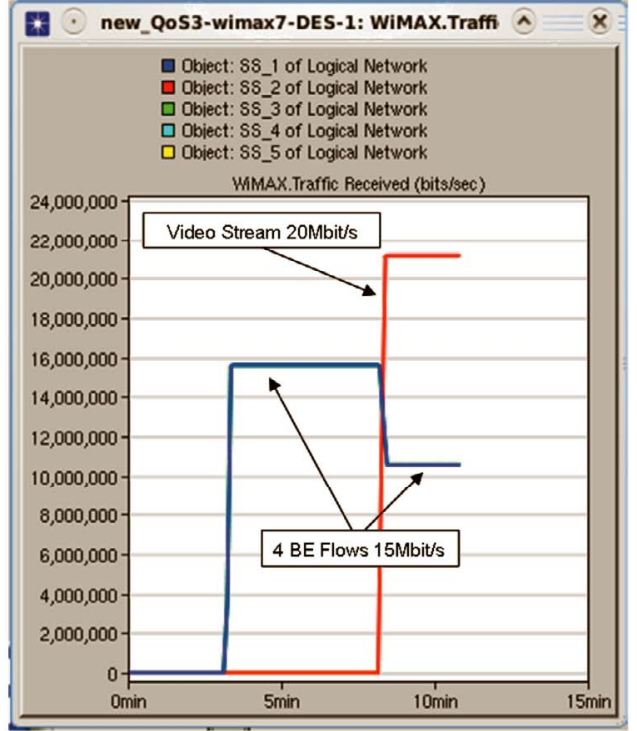

Figure 2. Traffic received from WiMAX Terminals.

\section{INTEGRATION WIMAX-EPON}

Here, we consider the integration between WiMAX network and Ethernet PON; this is one of the possibilities to cover, with broadband access techniques, areas where it is difficult, in economic and deployment terms, to install the optical fibre, e. g. in mountainous zones. In particular, we consider the BS as an Optical Network Unit (ONU) of EPON network (Fig. 3). In this case, it is necessary to manage the QoS in all network's segments, that is in WiMAX network (by procedures allowed in the standard)[3], in EPON (by definition of VLANs and relative CoSs, dependently on the bases of the service carried) and in the core network (by DiffServ over MPLS technique).

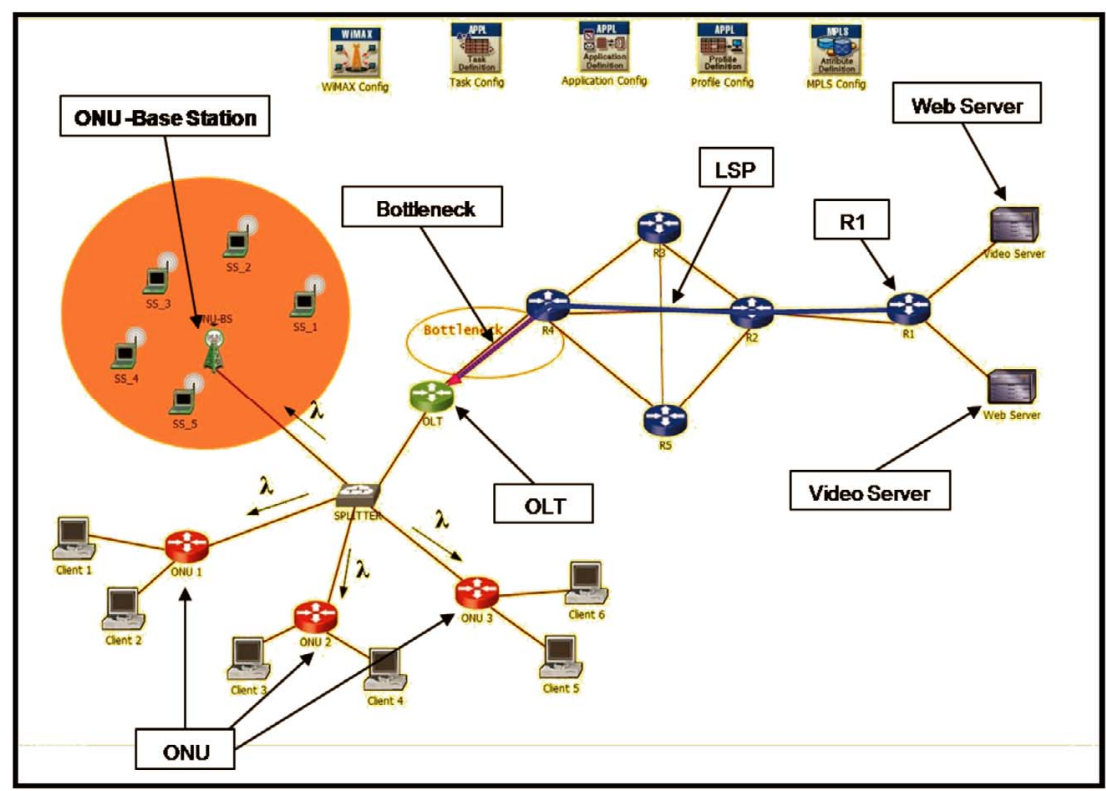

Figure 3. BS connected to ONU EPON. 
Several congestion scenarios have been considered, but for sake of brevity we report only the case in which there is a saturation before WiMAX network. EPON is simulated in OPNET with a multipoint access network. In this case, QoS is managed by MPLS/VLAN tagging. The congestion starts after 8 minutes and it lasts about 2 mins. Fig. 4 shows the traffic received from the WiMAX terminals. Each Web Flow carries about 5 Mbit/s, whilst the High Priority flow carries multiple Video Streams (about $33 \mathrm{Mbit} / \mathrm{s}$ ). It can be seen that, when the congestion occurs, the throughput of Best Effort flows (Web Flows) experiences degradation, while the throughput of guaranteed QoS Flow (Video Stream) is not affected.

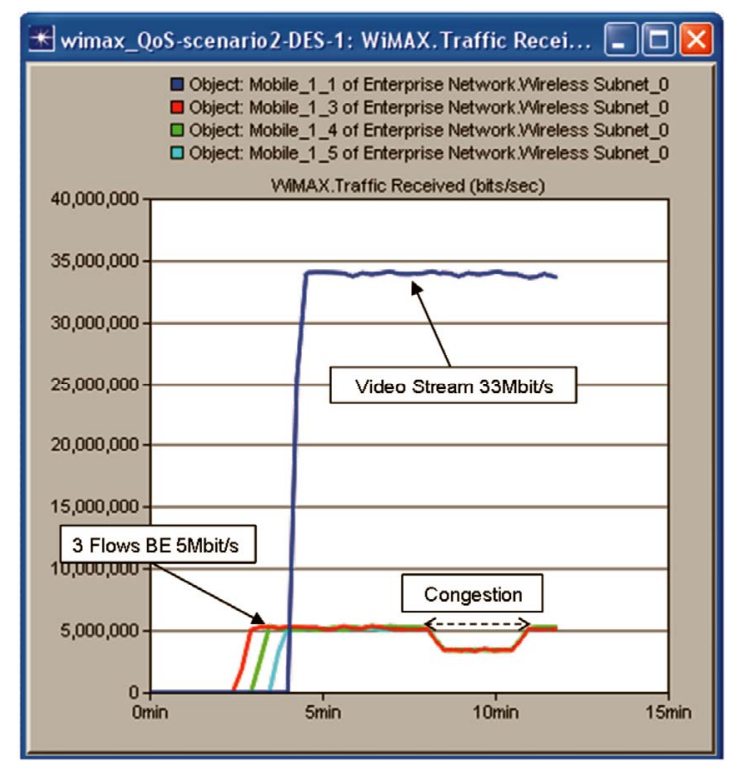

Figure 4. Traffic received with congestion on the link before WiMAX cell.

\section{INTEGRATION WIMAX-WDM-PON}

In the second scenario, it has been considered the integration between WiMAX network and WDM-PON network. In such context, each user (in this case the BS) has a distinct wavelength, thus removing the limitations imposed by the sharing of bandwidth. Figure 4 shows the simulation scenario.

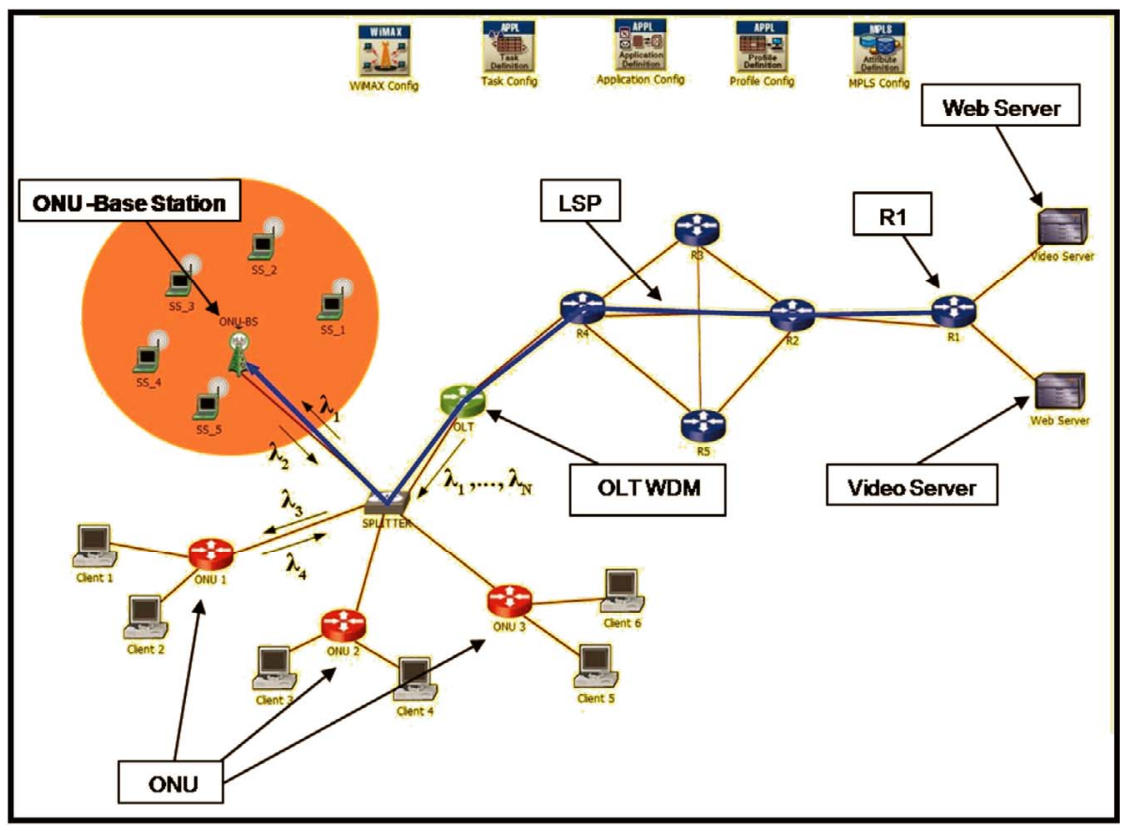

Figure 5. Base Station connects to ONU WDM-PON.

The core section of this network is represented by 4 core routers, the edge section is composed by a router R1, where are linked the Video Server and the Web Server, and the access sections consists of a WDM-PON, simulated in OPNET by a multipoint access network. In this case, congestion could occur in WiMAX cell. The network configuration considered corresponds to the typical case of a service running from a server to some 
users. QoS is managed by BS by five types of CoS: Unsolicited Grant Service (UGS), real-time Polling Service (rtPS), non-real-time Polling Service (nrtPS), Extended real-time Polling Service (ErtPS), Best Effort (BE). The priority traffic is assigned to service class rtPS configured to have $25 \mathrm{Mb} / \mathrm{s}$ of reserved bandwidth, in this way the BS assures this capability to the user that required it. In Fig. 6 shows the throughput of traffic on the WiMAX terminals: it can be seen how the traffic treated with the QoS control does not experience any effect, even if it is achieved the max capacity managed by the Base Station (about $70 \mathrm{Mbit} / \mathrm{s}$ ).

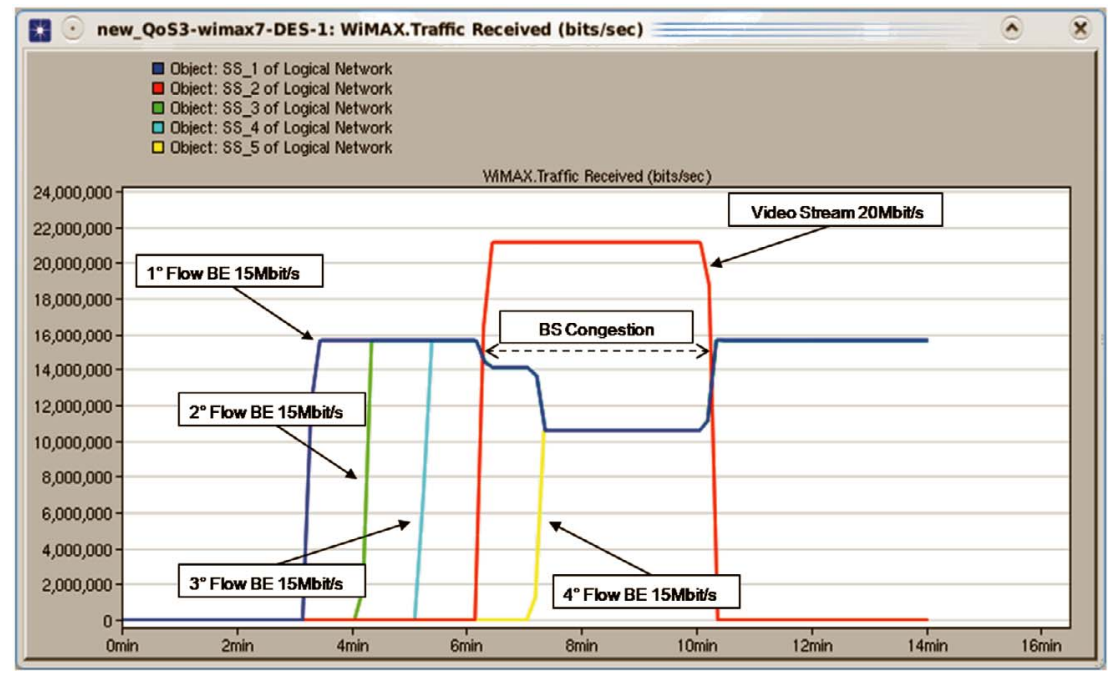

Figure 6. Traffic received from WiMAX terminals.

\section{CONCLUSIONS}

In this work three possible scenarios for integration of WiMAX in the optical telecommunication networks are shown, and we showed how it can be ensure the QoS of specific services by working both directly in the cell WiMAX and before of the same with techniques MPLS/VLAN tagging. It can be concluded that solutions with this type of integration can be used to provide the necessary bandwidth to services that require it.

\section{ACKNOWLEDGEMENTS}

The work described in this paper was carried out with the support of the BONE-project ("Building the Future Optical Network in Europe"), a Network of Excellence funded by the European Commission through the 7th ICT-Framework Programme.

The Authors thank OPNET for the licenses and the consultations on the OPNET simulation code.

\section{REFERENCES}

[1] S. Pompei, et al:: Quality of Service investigation on High Definition IPTV services in a Multivendor Optical Network with ADSL access, in Proc. of ICTON 2007, vol. 4, Rome, Italy, July 1-5, 2007.

[2] L. Hutcheson: FTTx: current status and the future, IEEE Communications Magazine, vol. 46, no. 7, pp. 90-95, Jul. 2008.

[3] IEEE Standard 802.16-2004: IEEE standard for Local Metropolitan Area Networks - Part16: Air Interface for Fixed Broadband Wireless Access Systems, Oct. 2004

[4] G. Shen, et al.: Fixed mobile convergence architecture for broadband access: Integration of EPON and WiMAX, IEEE Communication Magazine, vol. 45, no. 8 pp. 44-50, Aug. 2007. 